\title{
Samambaias e licófitas da Mata do Buraquinho, Paraíba, Brasil
}

\author{
Augusto César Pessôa Santiago ${ }^{1 \%}$ \\ Maria Alves de Sousa ${ }^{2}$ \\ Eva de Sousa Santana (in memorian) ${ }^{3}$ \\ Iva Carneiro Leão Barros ${ }^{4}$ \\ ${ }^{1}$ Laboratório de Biodiversidade, Núcleo de Biologia, Centro Acadêmico de Vitória \\ Universidade Federal de Pernambuco \\ Rua Alto do Reservatório, Bela Vista, CEP 55608-680, Vitória de Santo Antão - PE, Brasil \\ ${ }^{2}$ Universidade Federal da Paraíba, João Pessoa - PB, Brasil \\ ${ }^{3}$ Universidade Federal de Pernambuco, Recife - PE, Brasil \\ ${ }^{4}$ Laboratório de Pteridófitas, Departamento de Botânica, Centro de Ciências Biológicas \\ Universidade Federal de Pernambuco, Recife - PE, Brasil \\ * Autor para correspondência \\ augustosantiago@yahoo.com.br
}

Submetido em 15/07/2013

Aceito para publicação em 06/01/2014

\section{Resumo}

A Mata do Buraquinho é um remanescente de Mata Atlântica na região Nordeste do Brasil, em área urbana. $\mathrm{O}$ estudo foi baseado em coletas realizadas pelos autores ao longo das três últimas décadas e no levantamento dos principais herbários no Nordeste brasileiro. Foram registradas 22 espécies, uma licófita e 21 samambaias (distribuídas em 16 gêneros e 10 famílias). A maioria das espécies é amplamente distribuída no território americano e nas regiões brasileiras, algumas com poucos registros na área ocupada pela Mata Atlântica nordestina: Psilotum nudum (L.) P. Beauv., Ceratopteris thalictroides (L.) Brongn. e Actinosthachys subtrijuga (Mart.) C. Presl. Em relação aos aspectos ecológicos, a maioria das espécies se apresentou como herbácea, terrícola e hemicriptófita, ocorrendo no interior dos fragmentos. Em relação ao uso potencial, mais de $70 \%$ das espécies apresentam alguma indicação medicinal, ornamental ou alimentícia. Apesar do número reduzido de espécies, a flora de samambaias e licófitas da Mata do Buraquinho apresenta riqueza expressiva no contexto estadual, com espécies que ressaltam a importância dessa flora e a necessidade de sua preservação.

Palavras-chave: Florística; Mata Atlântica; Pteridófitas

\section{Abstract}

Ferns and lycophyta from Mata do Buraquinho, Paraíba, Brazil. Mata do Buraquinho is a remnant of Atlantic Forest in the Northeast region of Brazil, in an urban area. The study was based on collection made by the authors over the last three decades and on the survey of the major herbaria in northeastern Brazil. We registered 22 species, 1 lycophyte and 21 ferns (distributed into 16 genera and 10 families). Most species are widely distributed in the American territory and in the Brazilian regions, some of them with few records in the area occupied by the northeastern Atlantic Forest: Psilotum nudum (L.) P. Beauv., Ceratopteris thalictroides (L.) Brongn. and Actinosthachys subtrijuga (Mart.) C. Presl. Regarding the ecological aspects, most species showed up as herbaceous, terrestrial, and hemicryptophyte, occurring within the fragments. Regarding the potential use, 
more than $70 \%$ of the species have some medicinal, ornamentation, or food indication. Despite the small number of species, the fern and lycophyte flora in Mata do Buraquinho has a significant richness in the state context, with species highlighting the importance of this flora and the need for its preservation.

Key words: Atlantic Forest; Floristics; Pteridophytes

\section{Introdução}

As samambaias e licófitas representam as duas linhagens evolutivas das plantas vasculares sem sementes, anteriormente agrupadas sob o termo "pteridófitas" (SMITH et al., 2006). Considerando os dois grupos, segundo Moran (2008), são conhecidas cerca de 13.600 espécies, das quais 3.500 ocorrem na América do Sul. No Brasil são registradas 1.208 espécies, onde se destaca o domínio fitogeográfico da Floresta Atlântica, com mais de 830 registros (PRADO; SYLVESTRE, 2012).

A Floresta Atlântica possui reconhecida importância na biodiversidade brasileira e se estende do Ceará ao Rio Grande do Sul, sendo, muitas vezes, subdividida em centros de endemismo, já que é bastante heterogênea, com grande variação latitudinal e altitudinal (PRANCE, 1982; GALINDO-LEAL; CÂMARA, 2004). Na porção da Floresta Atlântica ao norte do Rio São Francisco, conhecida como Floresta Atlântica Nordestina, estimase cerca de 300 espécies de samambaias e licófitas (PAULA-ZÁRATE, 2004; SANTIAGO, 2006 e dados de herbário). Esta região compreende os remanescentes localizados entre os estados de Alagoas e Rio Grande do Norte, incluindo os encraves localizados no Ceará, e é a região mais devastada da Floresta Atlântica (TABARELLI et al., 2006).

Na Floresta Atlântica Nordestina, os estados de Pernambuco e Ceará destacam-se em relação ao estudo das samambaias e licófitas, enquanto nos estados de Alagoas, Paraíba e Rio Grande do Norte os estudos são incipientes (BARROS, 1996; BARROS et al., 2006) e merecem atenção para possíveis ações conservacionistas, que incluam o conhecimento da riqueza destes grupos, bem como o conhecimento de seus potenciais econômicos (SYLVESTRE, 2002).

Para o estado da Paraíba, são citadas 42 espécies de samambaias e quatro licófitas (PRADO; SYLVESTRE,
2012). O estado carece de trabalhos com os grupos, sendo encontrados alguns com registros de espécies (SOUSA; OLIVEIRA, 1996; FÉLIX et al., 1996; SOUSA et al., 2001; 2002) e citações em obras de maior abrangência (LUETZELBURG, 1922/1923; ALSTON, 1936; ANDRADE LIMA, 1969; PONTUAL, 1971; HIRAI; PRADO, 2000; SALINO; SEMIR, 2002; 2004; FERNANDES, 2003). Recentemente foram publicados alguns levantamentos florísticos para o Estado. Barbosa et al. (2012) citam 24 espécies de samambaias e duas licófitas, para a Reserva Biológica de Guaribas. Dentre as espécies citadas nove não são registradas na Lista da Flora do Brasil. Barros e Xavier (2013) citam 13 espécies para o Parque Estadual Mata do Xém-Xém e Lourenço e Xavier (2013) citam 14 espécies para a Estação Ecológica do Pau-Brasil, destacando a primeira ocorrência de Marsilea minuta L. para a Paraíba. Estes trabalhos totalizam o registro de 56 espécies de "pteridófitas" para o estado.

Desta forma, este trabalho teve o objetivo de apresentar uma listagem das espécies de samambaias e licófitas encontradas na Mata do Buraquinho, importante remanescente de Floresta Atlântica em área urbana, bem como discutir seus aspectos ecológicos, distribuição geográfica e potencial econômico.

\section{Material e Métodos}

\section{Área de estudo}

A Mata do Buraquinho $\left(07^{\circ} 06^{\prime} \mathrm{S}\right.$ e $\left.34^{\circ} 52^{\prime} \mathrm{W}\right)$ é um remanescente de Floresta Atlântica, inserido no município de João Pessoa, Paraíba, sendo um dos principais vestígios desta Floresta no Estado (ANDRADE LIMA; ROCHA, 1971). Segundo dados do posto do Instituto Nacional de Meteorologia, localizado na capital paraibana, a altitude local é de $45 \mathrm{~m}$ e a precipitação pluviométrica chega a $2.000 \mathrm{~m}$ anuais. $\mathrm{O}$ 
clima é quente e úmido, com temperatura média anual de $25^{\circ} \mathrm{C}$ e umidade atmosférica em torno de $80 \%$ (LIMA; HECKENDORFF, 1985).

A mata foi dividida durante a construção do Campus I da Universidade Federal da Paraíba e da BR 232, restando um fragmento contínuo de 471 ha e outros pequenos fragmentos dentro do Campus Universitário (BARBOSA, 2008; DIAS et al., 2006). Atualmente estão instaladas no local as dependências do Jardim Botânico Benjamim Maranhão.

\section{Coleta e análise das espécies}

O presente trabalho é baseado em coletas realizadas nas décadas de 80 e 90, por M.A. Sousa e E.S. Santana e por duas coletas do primeiro autor no ano de 2004, onde foram feitas visitas de três dias na estação chuvosa e três dias na estação seca. Também foram visitados os principais herbários dos estados da Paraíba (EAN e JPB) e Pernambuco (UFP, PEUFR e IPA). O material testemunho está depositado nas coleções do Herbário da Universidade Federal da Paraíba (JPB) e da Universidade Federal de Pernambuco (UFP).

Neste trabalho, foram adotados os termos samambaias e licófitas, assim como sugerido por Smith et al. (2008). O sistema de classificação adotado é o proposto por Smith et al. (2006), para as samambaias, e, para as licófitas, segue-se a circunscrição de gêneros proposta por Øllgaard (2012). Os nomes dos autores das espécies foram abreviados segundo Pichi-Sermolli (1996). Nas tabelas, são apresentadas as duas linhagens evolutivas, onde as famílias estão dispostas em ordem alfabética.

\section{Distribuição geográfica e aspectos ecológicos}

Para a análise da distribuição geográfica no mundo, seguiu-se a classificação utilizada por Gasper et al. (2012), baseada em Parris (2001) e Moran e Smith (2001), onde foram considerados os padrões: introduzidas (espécie introduzida do Velho Mundo nas Américas e hoje de ocorrência subespontânea), pantropicais (ocorrem em áreas tropicais do Novo e do
Velho Mundo), cosmopolitas (ocorrem no Velho e no Novo Mundo, excedendo o limite tropical), americanas (espécies ocorrentes na América do Sul, América Central e eventualmente na América do Norte), sul-americanas (espécies restritas aos países da América do Sul).

Os aspectos ecológicos analisados (habitat, hábito, formas de vida e locais de ocorrência) foram baseados em Ambrósio e Barros (1997), Santiago e Barros (2003) e Santiago et al. (2004). Abaixo são informadas as classificações das espécies de acordo com cada aspecto observado:

Hábito: herbácea (planta com caule macio ou maleável, normalmente rasteiro, sem lignina ou pouco lignificado) e trepadeira (espécie que apresenta crescimento inicial no solo e posteriormente usa outro vegetal como suporte).

Habitat: corticícola (espécie que cresce e se desenvolve sobre outro vegetal), terrícola (espécie que cresce e se desenvolve no solo), rupícola (espécie que vive sobre a pequena camada de húmus e terra nas rochas) e aquática (espécie que vive como flutuante em rio ou açude).

Formas de vida: hemicriptófita (espécies que possuem gema de renovação situada na superfície do solo ou ligeiramente abaixo deste), hidrófita (planta aquática flutuante), epífita (espécie que cresce e se desenvolve sobre outro vegetal) e helófita (espécie de ambientes encharcados, fixa no solo e com parte superior se desenvolvendo fora da água).

A importância econômica das espécies foi baseada nos trabalhos de Banerjee e Sen (1980), Barros e Andrade (1997), Santos e Sylvestre (2000; 2006), Santos et al. (2004) e alguns casos de observação pessoal dos autores.

Os locais de ocorrência foram considerados de acordo com os pontos de coleta dos diversos espécimes, tais como: açude, próximo ao açude (na margem do açude ou até cerca de $2 \mathrm{~m}$ de distância deste), clareira (área aberta pela queda de árvore), borda de mata (considerada do limite externo da mata até $20 \mathrm{~m}$ além deste), interior da mata (área considerada a partir de 20 $\mathrm{m}$ da borda), barranco (áreas com solo apresentando inclinação destacada do entorno), locais paludosos (locais constantemente encharcados pela água da chuva 
ou escoamento do regato), locais abertos (áreas próximas a mata, expostas ao sol).

\section{Resultados e Discussão}

Em relação à riqueza florística das samambaias ocorrentes na Mata do Buraquinho, foram registradas 21 espécies, distribuídas em 16 gêneros e 10 famílias (Tabela 1). A divisão Lycophyta foi representada apenas pela espécie Palhinhaea cernua Vasc. \& Franco
(Lycopodiaceae). Do total de 22 espécies, quatro são citadas pela primeira vez para o estado da Paraíba (Tabela 1). Assim, o número de samambaias e licófitas registradas para o estado da Paraíba é atualizado para 60 espécies. Contudo, segundo os dados observados nos herbários visitados, são estimadas cerca de 80 espécies de samambaias e licófitas para a Paraíba, sendo o presente trabalho expressivo na flora local, pois representa $1 / 4$ do observado para o Estado. Porém, acredita-se que este número de 80 espécies esteja bem

TABELA 1: Riqueza de espécies de samambaias e licófitas da Mata do Buraquinho (Jardim Botânico Benjamim Maranhão e Campus UFPB), estado da Paraíba, Brasil. ${ }^{1}$ Citadas pela primeira vez para o estado da Paraíba.

\section{TÁXONS Material Selecionado}

\section{LICÓFITA}

\section{Lycopodiaceae}

Palhinhaea cernua (L.) Vasc. \& Franco

\section{SAMAMBAIAS}

\section{Blechnaceae}

Blechnum serrulatum Rich.

\section{Lomariopsidaceae}

Nephrolepis biserrata (Sw.) Schott

Lygodiaceae

Lygodium venustum $\mathrm{Sw}$.

L. volubile $\mathrm{Sw}$.

Marsileaceae

Marsilea minuta L.

\section{Polypodiaceae}

Microgramma vacciniifolia (Langsd. \& Fisch.) Copel. Phlebodium decumanum (Willd.) J. Sm.

Serpocaulon triseriale (Sw.) A. R. Sm.

\section{Psilotaceae}

Psilotum nudum (L.) P. Beauv.

\section{Pteridaceae}

Acrostichum danaeifolium Langsd. \& Fisch. Ceratopteris thalictroides (L.) Brongn. ${ }^{1}$

Pityrogramma calomelanos (L.) Link

Pteris tripartita $\mathrm{Sw}$.

Pteris vittata L. $^{1}$

Vittaria lineata $\mathrm{L}$.

\section{Salviniacaeae}

Salvinia auriculata Aubl.

\section{Schizaeaceae}

Actinosthachys subtrijuga (Mart.) C. Presl. ${ }^{1}$

\section{Thelypteridaceae}

Macrothelypteris torresiana (Gaudich.) Ching ${ }^{1}$

Thelypteris dentata (Forsk.) E.P.St. John

T. interrupta (Willd.) K. Iwats.

T. serrata (Cav.) Alston
E.S. Santana 60 (PEUFR)

M.A. Sousa 736 (JPB), E.S. Santana 61 (PEUFR)

A. Santiago . 736 (UFP), E.S. Santana 84 (PEUFR)

E.S. Santana 69 (PEUFR)

E.S. Santana 62 (PEUFR)

P.C. Gadelha Neto 3143 (JPB)

E.S. Santana 95 (PEUFR), Santiago et al. 737 (JPB)

M.A. Sousa 735 (JPB), E.S. Santana 77 (PEUFR)

M.A. Sousa 940 (JPB), E.S. Santana 65 (PEUFR)

M.A. Sousa \& I. Oliveira 3378 (JPB)

E.S. Santana 59 (PEUFR)

A. Santiago et al. 733 (JPB)

M.A. Sousa 735 (JPB), E.S. Santana 105 (PEUFR)

A. Santiago et al. 730 (JPB, SP)

A. Santiago et al. 739 (JPB)

E.S. Santana 67 (PEUFR), A. Santiago et al. 743 (JPB)

E.S. Santana 65 (PEUFR)

L.P. Félix s/n (UFP)

A. Santiago . s/n (JPB)

A. Santiago et al. 735 (JPB),

M.A. Sousa 738 (JPB), A. Santiago et al. 740 (UFP)

M.A. Sousa 739 (JPB), A. Santiago et al. 742 (JPB) 
abaixo do real, pela escassez de trabalhos florísticos direcionados aos grupos.

O número de 22 espécies registradas neste estudo é relativamente baixo se comparado às localidades mais representativas, considerando a riqueza dos dois grupos, na Floresta Atlântica Nordestina (Tabela 2). Contudo, alguns fragmentos apresentam números inferiores ou pouco superiores ao registrado no presente trabalho (Tabela 2).

As famílias mais representativas neste estudo foram Pteridaceae, Thelypteridaceae e Polypodiaceae com seis, quatro e três espécies, respectivamente. As famílias mais representativas podem ser consideradas cosmopolitas e com um grande número de espécies, sendo que Pteridaceae e Polypodiaceae apresentam grande diversidade de formas entre seus representantes (TRYON; TRYON, 1982). Estas famílias aparecem como as mais numerosas em diversos trabalhos na Floresta Atlântica Nordestina, bem como na região como um todo (SANTIAGO et al., 2004; XAVIER; BARROS, 2005; SANTIAGO, 2006; PEREIRA et al., 2011). Contudo, destaca-se a ausência de espécies da família Dryopteridaceae, que é muito rica na região (SANTIAGO, 2006).

Em relação aos gêneros se destacam Thelypteris com três espécies, Pteris e Lygodium, com duas espécies cada. O gênero Thelypteris também ocorre como mais representativo em outros levantamentos dos grupos, como pode ser visto em trabalhos na região, citados anteriormente. Apesar da maioria das espécies deste gênero ser terrícola, seus representantes ocorrem desde áreas abertas expostas ao sol até locais paludosos no interior da mata. Thelypteris é o segundo gênero mais representado no Estado, com quatro espécies, superado apenas por Anemia, com seis espécies (PRADO; SYLVESTRE, 2012). Contudo nenhuma espécie de Anemia foi encontrada na área, sendo mais observadas em áreas mais afastadas do litoral e normalmente com altitudes superiores a $500 \mathrm{~m}$ (dados de herbário), como é o caso de algumas áreas consideradas como Brejos de Altitude no Estado. Outro gênero que é bem representado na Floresta Atlântica Nordestina (SANTIAGO, 2006) e que não está presente na área é Adiantum. Xavier e Barros (2005) associam a presença de espécies do gênero a áreas alteradas, o que seria o caso da Mata do Buraquinho que apresenta alto nível de pressão antrópica, evidenciado em seu histórico (BARBOSA, 2008).

Em relação à distribuição geográfica, quatro espécies (Macrothelypteris torresiana, Thelypteris dentata, Pteris tripartita e Pteris vittata) foram consideradas como introduzidas em território brasileiro. Estas espécies são amplamente distribuídas no Brasil e encontradas em muitas localidades na Região Nordeste (SALINO; SEMIR, 2002; PRADO; WINDISCH, 2000; SANTIAGO; BARROS, 2003; PRADO; SYLVESTRE,

TABELA 2: Riqueza de samambaias e licófitas em alguns remanescentes da Floresta Atlântica Nordestina.

\begin{tabular}{cccc} 
Fragmentos & $\begin{array}{c}\text { No de táxons } \\
\text { Infra-genéricos }\end{array}$ & $\begin{array}{c}\text { Área } \\
\text { (ha) }\end{array}$ & Referências \\
\hline Mata do Buraquinho (PB) & 22 & 400 & presente trabalho \\
Mata do Pau Ferro (PB) & 15 & 600 & Barros et al. (dados não publicados) \\
Reserva de Caetés (PE) & 16 & 157 & Farias et al. (1992), Barros et al. (1995/1996) \\
Refúgio Charles Darwin (PE) & 21 & 50 & Santiago e Barros (2003) \\
Reserva de Jangadinha (PE) & 25 & 85 & Ambrósio e Barros (1997) \\
REBIO Guaribas (PB) & 25 & 2.000 & Barbosa et al. (2012) \\
Serra Negra de Bezerros (PE) & 45 & 200 & Barros (1998) \\
Parque de Dois Irmãos (PE) & 43 & 387 & Pereira et al. (2011) \\
Reserva de Gurjaú (PE) & 77 & 745 & Pietrobom e Barros (2006) \\
Mata Maria Maior (AL) & 76 & 600 & Paula-Zárate et al. (2007) \\
Serra do Baturité (CE) & 94 & 32.600 & Pietrobom e Barros (2003) \\
Mata do Estado (PE) & 96 & 600 & Santiago et al. (2004) \\
Serra dos Macacos (PE) & 98 & 150 & Lopes et al. (dados não publicados) \\
RPPN Frei Caneca (PE) & 141 & 1.000 &
\end{tabular}


2012). De acordo com Johnson (1986), Marsilea minuta provavelmente é uma espécie introduzida no novo mundo, devido aos seus poucos e recentes registros. Thelypteris interrupta pode ser considerada cosmopolita e Actinosthachys subtrijuga é restrita a América do Sul. Cinco espécies (Nephrolepis biserrata, Palhinhaea cernua, Psilotum nudum, Pityrogramma calomelanos e Vittaria lineata) foram registradas como pantropicais. As outras espécies, constituindo a maioria, se enquadram como americanas, sendo amplamente distribuídas pela região Neotropical. De um modo geral, as espécies amplamente distribuídas na região Neotropical, compõem a principal parcela destes grupos (samambaias e licófitas) na Floresta Atlântica Nordestina, sendo poucas as espécies registradas como endêmicas ao Brasil e sem a presença de endemismos regionais (SANTIAGO, 2006).

Apesar da maioria das espécies apresentar ampla distribuição no território brasileiro (PRADO; SYLVESTRE, 2012), algumas merecem destaque, pela ocorrência restrita, considerando o limite da Floresta Atlântica Nordestina (sensu PRANCE, 1982), como é o caso de Psilotum nudum, Ceratopteris thalictroides e Actinosthachys subtrijuga. Psilotum nudum é registrado também para o estado de Pernambuco, ocorrendo em apenas uma localidade, na Mata de Dois Irmãos, Recife (BARROS; SANTIAGO, 2007). Em visitas recentes realizadas para a Mata de Dois Irmãos não foi feito nenhum registro desta espécie. Sendo assim, esta espécie pode ser considerada potencialmente ameaçada de extinção local. Actinosthachys subtrijuga também só é registrada em mais uma localidade, na referida floresta, para a área do Refúgio Ecológico Charles Darwin em Pernambuco (SANTIAGO; BARROS, 2003). Esta espécie figura na lista da Flora ameaçada de extinção do Rio Grande do Sul (Decreto Estadual 42.09901/01203). Ceratopteris thalictroides também não é bem representada nos herbários da região, indicando poucos pontos de registro, ocorrendo nos municípios de Pombos e Camocim de São Félix em Pernambuco. Apesar de ser considerada introduzida, Marsilea minuta é encontrada no Brasil apenas nos estados de Pernambuco, Rio de Janeiro (WINDISCH, 2013) e Paraíba (LOURENÇO; XAVIER, 2013).
Em relação ao hábito a maioria das espécies é herbácea, diferindo apenas as duas espécies de Lygodium, que se comportam como trepadeiras. Em relação ao habitat as espécies registradas como terrícolas representaram $60 \%$, seguidas pelas corticícolas $(22 \%)$, aquáticas (14\%) e rupícolas (4\%) (Tabela 3).

De acordo com as formas de vida, as espécies foram registradas como hemicriptófitas (60\%), epífitas (22\%), helófitas $(9 \%)$ e hidrófitas $(9 \%)$. De um modo geral as espécies foram mais coletadas no interior da mata, com (60\%), superando as de borda $(18 \%)$, porém algumas se mostraram indiferentes (22\%) (Tabela 3 ). Estes padrões são observados em muitos trabalhos com samambaias e licófitas na região (PIETROBOM; BARROS, 2003; SANTIAGO et al., 2004; BARROS et al., 2006; PEREIRA et al., 2011).

Das espécies registradas, 16 apresentam potencial econômico (Tabela 3), principalmente medicinal e ornamental. Apesar dos registros de utilização destas plantas na medicina popular (BARROS; ANDRADE, 1997; SANTOS; SYLVESTRE, 2000; 2006) e na comprovada atividade antibiótica de algumas espécies (BANERJEE; SEN, 1980), ainda são poucos os trabalhos que enfocam esta abordagem. Algumas espécies como as do gênero Lygodium são utilizadas em rituais místicos de onde vem seu nome popular de "abre-caminho" (ALBUQUERQUE et al., 1997).

Sem dúvidas, o potencial econômico mais explorado nas samambaias e licófitas é o ornamental. No presente trabalho, doze espécies já foram observadas na Região Nordeste sendo utilizadas para este fim. Nephrolepis biserrata é fácil de cultivar em residências e apresenta grande beleza e Psilotum nudum, apresenta potencial de cultivo, visto que muitas vezes é observado crescendo em ambientes alterados e por vezes em jardins (observação dos autores). Salvinia auriculata é normalmente utilizada em tanques d'água e aquários. Pahlinhaea cernua é bastante utilizada em arranjos ornamentais. Durante coletas na Mata do Buraquinho na década de 90 e coletas recentes no Parque de Dois Irmãos (Pernambuco), foi possível observar a extração de indivíduos desta espécie por populares para este propósito. 
TABELA 3: Aspectos ecológicos e potencial econômico das samambaias e licófitas ocorrentes na Mata do Buraquinho (João Pessoa, Paraíba, Brasil). Hábitat: $\mathrm{C}=$ Corticícola, $\mathrm{T}=$ Terrícola, $\mathrm{R}=$ Rupícola, $\mathrm{A}=$ Aquática. Locais de ocorrência: $\mathrm{AC}=$ Açude, $\mathrm{CL}=$ Clareira, $\mathrm{BM}=$ Borda de Mata, $\mathrm{BR}=$ Barranco, $\mathrm{IM}=$ Interior da mata, $\mathrm{LA}=$ Locais abertos, $\mathrm{LP}=$ Locais paludosos, $\mathrm{MR}=$ Margem de Regato, $\mathrm{PA}=$ Próximo ao açude. Formas de vida: $\mathrm{EP}=$ Epífita, $\mathrm{HC}=$ Hemicriptófita, $\mathrm{HI}=$ Hidrófita, $\mathrm{HL}=$ Helófita. Potencial Econômico: $\mathrm{A}=$ Alimentício, $\mathrm{M}=$ Medicinal, $\mathrm{O}=$ Ornamental.

\begin{tabular}{|c|c|c|c|c|}
\hline Táxons & Hábitat & $\begin{array}{l}\text { Formas } \\
\text { de Vida }\end{array}$ & $\begin{array}{c}\text { Locais de } \\
\text { Ocorrência }\end{array}$ & $\begin{array}{c}\text { Potencial } \\
\text { Econômico }\end{array}$ \\
\hline \multicolumn{5}{|l|}{ LICÓFITA } \\
\hline Palhinhaea cernua & $\mathrm{T}$ & $\mathrm{HC}$ & BM, BR, LP & $\mathrm{O}, \mathrm{M}$ \\
\hline \multicolumn{5}{|l|}{ SAMAMBAIAS } \\
\hline Acrostichum danaeifolium & $\mathrm{T}$ & $\mathrm{HL}$ & IM, BM, LA, LP & $\mathrm{O}, \mathrm{M}$ \\
\hline Blechnum serrulatum & $\mathrm{T}$ & $\mathrm{HC}$ & IM, BM, LA, LP & $\mathrm{M}$ \\
\hline Ceratopteris thalictroides & A & $\mathrm{HL}$ & IM, LP, MR & $\mathrm{O}, \mathrm{A}$ \\
\hline Lygodium venustum & $\mathrm{T}$ & $\mathrm{HC}$ & IM, BM & $\mathrm{M}, \mathrm{O}$ \\
\hline Lygodium volubile & $\mathrm{T}$ & $\mathrm{HC}$ & $\mathrm{BM}, \mathrm{MR}, \mathrm{LP}$ & $\mathrm{M}, \mathrm{O}$ \\
\hline Macrothelypteris torresiana & $\mathrm{T}$ & $\mathrm{HC}$ & $\mathrm{IM}, \mathrm{MR}, \mathrm{BR}$ & M \\
\hline Marsilea minuta & A & $\mathrm{HI}$ & IM, LP & $\mathrm{O}$ \\
\hline Microgramma vacciniifolia & $\mathrm{C}$ & $\mathrm{EP}$ & IM, BM, LA & M \\
\hline Nephrolepis biserrata & $\mathrm{C}$ & EP & IM, LP & $\mathrm{O}$ \\
\hline Pityrogramma calomelanos & $\mathrm{T}$ & $\mathrm{HC}$ & IM, LP, MR, PA & $\mathrm{M}$ \\
\hline Phlebodium decumanum & $\mathrm{C}$ & $\mathrm{EP}$ & $\mathrm{IM}, \mathrm{CL}$ & $\mathrm{O}, \mathrm{M}$ \\
\hline Serpocaulon triseriale & $\mathrm{C}$ & $\mathrm{EP}$ & $\mathrm{IM}$ & $\mathrm{O}, \mathrm{M}$ \\
\hline Psilotum nudum & $\mathrm{T}$ & $\mathrm{HC}$ & IM, LP & $\mathrm{O}, \mathrm{M}$ \\
\hline Pteris tripartita & $\mathrm{T}$ & $\mathrm{HC}$ & IM, MR, LP & - \\
\hline Pteris vittata & $\mathrm{R}$ & $\mathrm{HC}$ & BM, MR & - \\
\hline Salvinia auriculata & A & HI & $\mathrm{IM}, \mathrm{AC}$ & $\mathrm{O}, \mathrm{M}$ \\
\hline Actinosthachys subtrijuga & $\mathrm{T}$ & $\mathrm{HC}$ & IM, LP & - \\
\hline Thelypteris dentata & $\mathrm{T}$ & $\mathrm{HC}$ & IM, BR, MR & $\mathrm{M}, \mathrm{O}$ \\
\hline Thelypteris interrupta & $\mathrm{T}$ & $\mathrm{HC}$ & $\mathrm{BM}, \mathrm{LA}, \mathrm{LP}$ & - \\
\hline Thelypteris serrata & $\mathrm{T}$ & $\mathrm{HC}$ & IM, BM, LP, PA & - \\
\hline Vittaria lineata & $\mathrm{C}$ & EP & IM, LP & - \\
\hline
\end{tabular}

Apenas uma espécie, Ceratopteris thalictroides, foi registrada como alimentícia, sendo utilizada em saladas em muitos países (JONES, 1987). O costume de utilizar samambaias na alimentação é pouco difundido em território nacional, mas pode ser observado em alguns locais como Belo Horizonte (MG) e Vitória (ES) (A. Santiago observação pessoal). Um gênero muito utilizado é Pteridium, bastante consumido no Japão e que possui comprovada atividade tóxica (MORAN, 2004). Christensen (1997) observou que tribos indígenas de Sarawak (Malásia) consomem muitas frondes jovens em sua alimentação, chegando algumas localidades a apresentar a utilização de 16 espécies.

O uso das samambaias e licófitas é bastante antigo, para os mais diversos fins, como pode ser observado em
Windisch (2002a) e Moran (2004). Contudo, segundo os autores, os usos tradicionais não representam ameaça as espécies e as maiores preocupações devem ser voltadas para a perda de habitat ocasionada pela devastação e fragmentação e também pela retirada de seu habitat natural para fins comerciais (ornamentais).

É grande a necessidade de trabalhos voltados para o conhecimento da biota na região da Floresta Atlântica Nordestina, já que esta área da Floresta Atlântica é uma das mais degradadas desde o tempo da colonização, além de ter a biodiversidade pouco conhecida (GALINDOLEAL; CÂMARA, 2004). Atualmente restam apenas cerca de 5\% de sua área original na região Nordeste, e os remanescentes são pequenos e isolados (RANTA et al., 1998; SILVA; TABARELLI, 2000), ocasionando 
provavelmente uma alta taxa de extinção local (e até o desaparecimento de espécies endêmicas) na fauna e na flora.

Para as samambaias e licófitas, a fragmentação e a perda de habitats são fatores diretamente relacionados com a ameaça da riqueza e diversidade dos grupos, já que a maioria das espécies ocorre no interior das florestas, associadas a microambientes úmidos e sombreados (BARROS et al., 2006). A Mata do Buraquinho inserida na porção da Floresta Atlântica Nordestina se destaca como um dos maiores remanescentes em área urbana da região. Esta Floresta ainda vem sendo drasticamente reduzida e o perigo de uma grande extinção de espécies é iminente (COIMBRA-FILHO; CÂMARA, 1996; SILVA; TABARELLI, 2000; GALINDO-LEAL; CÂMARA, 2004).

Este trabalho é um dos poucos levantamentos da flora de samambaias e licófitas em um remanescente de Floresta Atlântica no estado da Paraíba. O número de espécies registradas na Mata do Buraquinho é expressivo dentro do contexto regional, além de apresentar espécies de samambaias que ocorrem de forma restrita na Floresta Atlântica Nordestina. Essas informações são importantes, já que algumas espécies destes grupos podem ser consideradas como sensível às modificações ambientais e com possibilidade de ser utilizado em monitoramento ambiental. $\mathrm{O}$ acompanhamento das populações dos grupos estudados pode servir para observação de como os grupos estão respondendo ao processo de regeneração ou aumento da pressão antrópica na área. Outro aspecto importante é o potencial econômico apresentado pelas plantas registradas, o que pode servir para estudos científicos posteriores e planos de manejo com a população do entorno, que deve se sentir engajada na preservação deste remanescente florestal.

Iniciativas como a publicação de listas de organismos para um determinado local, contribuindo com o conhecimento sobre a distribuição geográfica destes, são essenciais para que possam ser tomadas medidas conservacionistas. $\mathrm{O}$ perigo de extinções locais deve ser considerado, já que podem acarretar em perda do patrimônio genético de populações disjuntas, como é observado por Windisch (2002b). A Mata do Buraquinho merece atenção devido à riqueza de espécies que ainda guarda, não só das samambaias e licófitas, mas de outros grupos da fauna e da flora (SANTANA et al., 2008; BARBOSA, 2008; A. VASCONCELLOS comunicação pessoal).

\section{Agradecimentos}

Os autores agradecem as Instituições que apoiaram o trabalho: Universidade Federal de Pernambuco, Universidade Federal da Paraíba, Jardim Botânico Benjamim Maranhão e a Fundação $O$ Boticário de Proteção à Natureza (pelo apoio financeiro nas coletas complementares, realizadas em 2004). Agradecemos também ao Dr. Iderval de Oliveira, Dr. Leonardo Pessoa Félix, Biólogo Pedro Gadelha Neto, Dra. Ma Socorro Pereira e Dr. Emerson Lucena, pelo apoio e companhia nas coletas; ao Dr. Jefferson Prado pela análise do material de Pteris tripartita; ao Biólogo Rafael Farias pelos comentários no manuscrito; ao Dr. Sergio Xavier pela identificação de Marsilea minuta.

\section{Referências}

ALBUQUERQUE, U. P.; CHIAPPETA, A. A.; BARROS, I. C. L. Pteridófitas utilizadas nos culto Afro-Brasileiros em Recife-PE. Biologica Brasilica, Recife, v. 7, n. 1/2, p. 23-31, 1997.

ALSTON, A. H. G. The Brazilian species of Selaginella. Reportorium Specierum Novarum Regni Vegetabilis, Berlin, v. 40, p. 303-319, 1936

AMBRÓSIO, S. T.; BARROS, I. C. L. Pteridófitas de uma área remanescente de Floresta Atlântica do Estado de Pernambuco, Brasil. Acta Botanica Brasilica, Feira de Santana, v. 11, n. 2, p. 105-113, 1997.

ANDRADE-LIMA, D. Pteridófitas que ocorrem nas floras Extra-Amazônicas e Amazônicas do Brasil e proximidades. In: CONGRESSO NACIONAL DE BOTÂNICA, 20, 1969, Goiânia. Anais... Goiânia: SBB, 1969. p. 34-39.

ANDRADE-LIMA, D.; ROCHA, M. G. Observações preliminares sobre a Mata do Buraquinho, João Pessoa, Paraíba. Anais do Instituto de Ciências Biológicas, Recife, v. 1, n. 1, p. 47-61, 1971.

BANERJEE, R. D.; SEN, S. P. Antibiotic activity of Pteridophytes. Economic Botany, St. Louis, v. 34, p. 284-298, 1980.

BARBOSA, M. R. V. Floristic composition of a remnant of Atlantic Coastal Forest in João Pessoa, Paraíba, Barsil. In: THOMAS, W. W. (Org.). The Atlantic Coastal Forest of Northeastern Brazil. New York: New York Botanical Garden Press, 2008. p. 39-457.

BARBOSA, M. R. V.; THOMAS, W. W.; ZÁRATE, E. L. P.; LIMA, R. B.; AGRA, M. F.; LIMA, I. B.; PESSOA, M. C. R.; LOURENÇO, A. R. L.; DELGADO JÚNIOR, G. C.; PONTES, R. A. S.; CHAGAS, E. C. O.; VIANA, J. L.; GADELHA NETO, P. 
C.; ARAÚJO, C. M. L. R.; ARAÚJO, A. A. M.; FREITAS, G. B.; LIMA, J. R. L.; SILVA, F. O.; VIEIRA, L. A. F.; PEREIRA, L. A.; COSTA, R. M. T.; DURÉ, R. C.; SÁ, M. G. V. Checklist of the vascular plants of the Guaribas Biological Reserve, Paraíba,Brasil. Revista Nordestina de Biologia, João Pessoa, v. 20, n. 2, p. 79106, 2012.

BARROS, I. C. L. Pteridófitas. In: SAMPAIO, E. V. S. B.; MAYO, S. J.; BARBOSA, M. R. V. (Ed.). Pesquisa botânica nordestina: progresso e perspectivas. Recife: Sociedade Botânica do Brasil, Seção Regional de Pernambuco, 1996. p. 111-121.

BARROS, I. C. L. Biodiversidade e ecologia das espécies de pteridófitas (avencas samambaias e plantas afins) da Reserva Ecológica de Dois Irmãos. In: MACHADO, I. C.; LOPES, A. V.; PÔRTO, K. C. (Org.). Reserva Ecológica de Dois Irmãos: estudos em um remanescente de Mata Atlântica em Área Urbana (Recife-Pernambuco-Brasil). Recife: Ed. Universidade Federal de Pernambuco, 1998. p 137-155.

BARROS, I. C. L.; ANDRADE, L. H. C. Pteridófitas medicinais (samambaias, avencas e plantas afins). Recife: Ed. Universitária da Universidade Federal de Pernambuco, 1997. 223 p.

BARROS, I. C. L.; FONSECA, E. R.; VALDEVINO, J. A.; PAULA, E. L. Contribuição ao estudo taxonômico das pteridófitas ocorrentes na Reserva Ecológica de Caetés (Paulista, PE) - Thelypteridaceae. Dryopteridaceae. Davalliaceae. Polypodiaceae. Lycopodiaceae. Boletim da Sociedade Broteriana, Coimbra, v. 67, p. 271-286, 1995/1996.

BARROS, I. C. L; SANTIAGO, A. C. P. Pteridófitas do estado de Pernambuco, Brasil: Psilotaceae. Bradea, Rio de Janeiro, v. 12, p. 17-23, 2007.

BARros, I. C. L.; SANTIAGO, A. C. P.; PEREIRA, A. F. N.; PIETROBOM, M. R. Pteridófitas. In: PORTO, K. C.; TABARELLI, M.; CORTEZ, J. A. (Org.). Áreas críticas para a conservação da biodiversidade no Centro de Endemismo Pernambuco. Brasília: MMA, 2006. p. 147-171.

BARROS, S. C. A.; XAVIER, S. R. S. Samambaias em remanescente de Floresta Atlântica Nordestina (Parque Estadual Mata do XémXém, Bayeux, Paraíba). Pesquisas: Botânica, Porto Alegre, v. 64, p. 207-224, 2013

CHRISTENSEN, H. Uses of ferns in two indigenous communities in Sarawak, Malaysia. In: JOHNS, R. J. (Ed.). Holttum Memorial Volume. Kew: Royal Botanic Gardens, 1997. p 177-192.

COIMBRA-FILHO, A. F.; CÂMARA, I. G. Os limites originais do bioma Mata Atlântica na Região Nordeste do Brasil. Rio de Janeiro: Fundação Brasileira para a Conservação da Natureza, 1996. $86 \mathrm{p}$.

DIAS, S.; CANDIDO, D. M.; BRESCOVIT, A. D. Scorpions from Mata do Buraquinho, João Pessoa, Paraíba, Brazil, with ecological notes on a population of Ananteris mauryi Lourenço (Scorpiones, Buthidae). Revista Brasileira de Zoologia, Curitiba, v. 23, n. 3, p. 707-710, 2006.

FARIAS, M. C. A.; BARROS, I. C. L.; BELO, M. A. M. Pteridófitas da Reserva Ecológica de Caetés, Paulista, Pernambuco. Boletim da Sociedade Broteriana, Coimbra, v. 65, n. 1, p. 149-162, 1992.

FÉLIX, L. P.; SOUSA, M. A.; OLIVEIRA, I. C. Pteridófitas do Herbário Prof. Jayme Coelho de Moraes (EAN), Areia - Paraíba, Brasil: I - Vittariaceae. Revista Nordestina de Biologia, João Pessoa, v. 11, n. 1, p. 57-71, 1996.
FERNANDES, I. Taxonomia dos representantes de Cyatheaceae do Nordeste Oriental do Brasil. Pesquisas Botânica, São Leopoldo, v. 53, p. 7-53, 2003.

GALINDO-LEAL, C.; CÂMARA, I. G. The Atlantic forest of South America. Biodiversity status, threats, and outlook. Washington: Island Press, 2004. 488 p.

GASPER, A. L.; SALINO, A.; VIBRANS, A. C.; SEVEGNANI, L.; VERDI, M.; KORTE, A.; SANTOS, A. S.; DREVECK, S.; CADORIN, T. J.; SCHMITT, J. L.; CAGLIONI, E. Pteridófitas de Santa Catarina: um olhar sobre os dados do Inventário Florístico Florestal de Santa Catarina, Brasil. Acta Botanica Brasilica, Feira de Santana, v. 26, n. 2, p. 421-434, 2012.

HIRAI, R. Y.; PRADO, J. Selaginellaceae Willk. no Estado de São Paulo. Revista Brasileira de Botânica, São Paulo, v. 23, n. 3, p. 313-339, 2000

JOHNSON, D. M. Systematics of the New World species of Marsilea (Marsileaceae). Systematic Botany Monographs, Wyoming, v. 11, p. 1-87, 1986.

JONES, D. L. Encyclopaedia of ferns. Melbourne: British Museum (Natural History), London and Lothian Publishing Company Pty. Ltd., 1987. 450 p.

LIMA, P. J.; HECKENDORFF, W D. Climatologia. In: GOVERNO DO ESTADO DA PARAÍBA (Ed.). Atlas geográfico do Estado da Paraíba. João Pessoa: Secretária de Educação, Ed. Grafset, 1985. p 34-43.

LOURENÇO, J. D. S.; XAVIER, S. R. S. Samambaias da Estação Ecológica do Pau-Brasil, Paraíba, Brasil). Pesquisas: Botânica, Porto Alegre, v. 64, p. 225-242, 2013

LUETZELBURG, P. Von. Estudo Botânico do Nordeste. Rio de Janeiro: Inspetoria Federal de Obras Contra as Secas, publicação 57(série IA), v. II, 1922/1923. 126 p.

MORAN, R. C. A natural history of ferns. Portland: Timber Press, 2004. 302 p.

MORAN, R. C. Diversity, biogeography, and floristics. In: RANKER, T. A.; HAUFLER, C. H. (Ed.). Biology and evolution of ferns and Lycophytes. Cambridge: Cambridge University Press, 2008. p. 367-394.

MORAN, R. C.; SMITH, A. R. Phytogeographic relationships between neotropical and African-Madagascan pteridophytes. Brittonia, New York, v. 53, n. 2, p. 304-351, 2001.

ØLLGAARD, B. New combinations in Neotropical Lycopodiaceae. Phytotaxa, Auckland, v. 57, p. 10-22, 2012.

PARRIS, B. S. Circum-Antarctic continental distribution patterns in pteridophyte species. Brittonia, New York, v. 53, n. 2, p. 270 283, 2001 .

PAULA-ZÁRATE, E. L. Florística e fitogeografia das Pteridófitas do Estado do Ceará, Brasil. 2004. 269 f. Tese (Doutorado em Botânica) - Universidade de São Paulo, São Paulo. 2004.

PAULA-ZÁRATE, E. L.; FIGUEIREDO, M. A.; BARROS, I. C. L.; ANDRADE, L. H. C. Diversidade de pteridófitas da serra do Baturité, Ceará. In: OLIVEIRA, T. S. O.; ARAÚJO, F. S. (Org.). Diversidade e conservação da biota na serra do Baturité. Fortaleza: Edições UFC, 2007. p. 163-183.

PEREIRA, A. F. N.; BARROS, I. C. L.; SANTIAGO, A. C. P.; SILVA, I. A. A. Florística e distribuição geográfica das samambaias e licófitas da Reserva Ecológica de Gurjau. Rodriguésia, Rio de Janeiro, v. 62, n. 1, p. 1-10, 2011. 
PICHI-SERMOLLI, R. E. G. Authors of scientific names in Pteridophyta. Kew: Royal Botanical Garden, 1996. 108 p.

PIETROBOM, M. R.; BARROS, I. C. L. Pteridófitas de um fragmento florestal na Serra do Mascarenhas, Estado de Pernambuco, Brasil. Insula, Florianópolis, v. 32, n. 1, p. 73-118, 2003.

PIETROBOM, M. R.; BARROS, I. C. L. Associações entre pteridófitas em dois fragmentos de Floresta Atlântica do Nordeste Brasileiro. Biotemas, Florianópolis, v. 19, n. 3, p. 15-26, 2006.

PONTUAL, I. B. Pteridófitas de Pernambuco e Alagoas (I). Anais do Instituto de Ciências Biológicas, Recife, v. 1, n. 1, p. 153-260, 1971.

PRADO, J.; SYLVESTRE, L. S. Pteridófitas. Lista de espécies da flora do Brasil. 2012. Disponível em: <http://floradobrasil.jbrj.gov. br/2012/FB000007>. Acesso em: 15 out. 2012.

PRADO, J.; WINDISCH, P. G. The genus Pteris L. (Pteridaceae) in Brazil. Boletim do Instituto de Botânica, São Paulo, v. 13, p. 103-199, 2000.

PRANCE, G. T. Forest Refuges: evidences from woody angiosperms. In: PRANCE, G. T. (Ed.). Biological diversification in the tropics. New York: Columbia University Press, 1982. p. 137-158.

RANTA, P.; BLOM, T.; NIEMELA, J.; JOENSUU, E.; SIITONEN, M. The fragmented Atlantic rain Forest of Brazil: size, shape and distribution os forests fragments. Biodiversity and Conservation, New York, v. 7, p. 385-403, 1998.

SALINO, A.; SEMIR, J. Thelypteridaceae (Polypodiophyta) do Estado de São Paulo: Macrothelypteris, Thelypteris subg. Cyclosorus e Thelypteris subg. Steiropteris. Lundiana, Belo Horizonte, v. 3, n. 1, p. 9-27, 2002.

SALINO, A.; SEMIR, J. Thelypteris subg. Meniscium (Thelypteridaceae - Pterophyta) no Estado de São Paulo, Brasil. Revista Brasileira de Botânica, São Paulo, v. 27, n. 1, p. 103-114, 2004.

SANTANA, G. G.; VIEIRA, W. L. S.; PEREIRA-FILHO, G. A.; DELFIM, F. R.; LIMA, Y. C. C.; VIEIRA, K. S. Herpetofauna em um fragmento de Floresta Atlântica no Estado da Paraíba, Região Nordeste do Brasil. Biotemas, Florianópolis, v. 21, n. 1, p. 75-84, 2008 .

SANTIAGO, A. C. P. Pteridófitas da Floresta Atlântica ao norte do Rio São Francisco: florística, biogeografia e conservação. 2006. 124 f. Tese (Doutorado em Biologia Vegetal) - Universidade Federal de Pernambuco, Recife. 2006.

SANTIAGO, A. C. P.; BARROS, I. C. L. Pteridoflora do Refúgio Ecológico Charles Darwin (Igarassu, Pernambuco, Brasil). Acta Botanica Brasilica, Feira de Santana, v. 17, n. 4, p. 597-604, 2003.

SANTIAGO, A. C. P.; BARROS, I. C. L.; SYlVESTRE, L. S. Pteridófitas ocorrentes em três fragmentos florestais de um brejo de altitude (Bonito, Pernambuco, Brasil). Acta Botanica Brasilica, Feira de Santana, v. 18, n. 4, p. 781-792, 2004.

SANTOS, M. G.; SYLVESTRE, L. S. Pteridófitas comercializadas por erveiros de Niterói e do Rio de Janeiro, RJ, Brasil: uma abordagem etnobotânica. Leandra, Rio de Janeiro, v. 15, p. 79-90, 2000 .

SANTOS, M. G.; SYLVESTRE, L. S. Aspectos florísticos e econômicos das pteridófitas de um afloramento rochoso do estado do Rio de Janeiro, Brasil. Acta Botanica Brasilica, Feira de Santana, v. 20, n. 1, p. 115-124, 2006.

SANTOS, M. G., SYlVESTRE, L. S.; ARAuJO, D. S. D. Análise florística das pteridófitas do Parque Nacional da Restinga de Jurubatiba, Rio de Janeiro, Brasil. Acta Botanica Brasilica, Feira de Santana, v. 18, n. 2, p. 271-280, 2004.

SILVA, J. M. C.; TABARELLI, M. Tree species impoverishment and the future flora of the Atlantic Forest of northeast Brazil. Nature, London, v. 404, p. 72-74, 2000.

SMITH, A. R.; PRYER, K. M.; SCHUETTPELZ, E.; KORAL, P.; SCHNEIDER, H.; WOLF, P. G. A classification for extant ferns. Taxon, Bratislava, v. 55, p. 705-731, 2006.

SMITH, A. R.; PRYER, K. M.; SCHUETTPELZ, E.; KORAL, P.; SCHNEIDER, H.; WOLF, P. G. Fern classification. In: RANKER, T. A.; HAUFLER, C. H. (Ed.). The biology and evolution of ferns and Lycophytes. Cambridge: Cambridge University Press, 2008. p. 417-467.

SOUSA, M. A.; OLIVEIRA, I. C. Psilotum nudum (L.) Beauv (Psilotaceae), primeiro registro para a Paraíba, Brasil. Revista Nordestina de Biologia, João Pessoa, v. 11, n. 1, p. 45-49, 1996.

SOUSA, M. A.; OLIVEIRA, I. C.; FÉLIX, L. P. Pteridófitas no Estado da Paraíba: Ophioglossaceae. Revista Nordestina de Biologia, João Pessoa, v. 16, n. 1/2, p. 23-26, 2002.

SOUSA, M. A.; OLIVEIRA, I. C.; SANTANA, E. S.; FÉLIX, L. P. Pteridófitas no Estado da Paraíba: Salviniaceae. Revista Nordestina de Biologia, João Pessoa, v. 15, n. 2, p. 11-16, 2001.

SYLVESTRE, L. S. Estudos taxonômicos e florísticos das pteridófitas brasileiras: desafios e conquistas. In: ARAÚJO, E. L.; MOURA, A. N.; SAMPAIO, E. V. S. B.; GESTINARI; L. M. S.; CARNEIRO, J. M. T. (Org.). Biodiversidade, conservação e uso sustentável da flora do Brasil. Vol. 1. Recife: Universidade Federal Rural de Pernambuco/Sociedade Botânica do Brasil, 2002. p. 194-195.

TABARELlI, M.; CORTEZ, J. A.; PORTO, K. C. Introdução. In: PORTO, K. C.; CORTEZ, J. A.; TABARELli, M. (Org.). Diversidade biológica e conservação da floresta Atlântica ao Norte do Rio São Francisco. Brasília: MMA, 2006. p. 15-18.

TRYON, R. M.; TRYON, A. F. Ferns and allies plants with special references to Tropical America. New York: SpringerVerlag, 1982. $875 \mathrm{p}$.

WINDISCH, P. G. Pteridófitas do Brasil: diversidade decrescente. In: ARAÚJO, E. L.; MOURA, A. N.; SAMPAIO, E. V. S. B.; GESTINARI, L. M. S.; CARNEIRO, J. M. T. (Org.). Biodiversidade, conservação e uso sustentável da flora do Brasil. Vol. 1. Recife: Universidade Federal Rural de Pernambuco/ Sociedade Botânica do Brasil, 2002a. p. 196-198.

WINDISCH, P. G. Fern conservation in Brazil. Fern Gazette, London, v. 16, n. 6, p. 295-300, 2002 b.

WINDISCH, P. G. Marsileaceae - Lista de espécies da flora do Brasil. 2013. Disponível em $<$ http://floradobrasil.jbrj.gov.br/jabot/ floradobrasil/FB91500>. Acesso em: 15 jun. 2013.

XAVIER, S. R. S.; BARROS, I. C. L. Pteridoflora e seus aspectos ecológicos ocorrentes no Parque Ecológico João Vasconcelos Sobrinho, Caruaru, PE, Brasil. Acta Botanica Brasilica, Feira de Santana, v. 19, n. 4, p. 775-781, 2005. 